\title{
The Mu2e Experiment at Fermilab
}

\author{
Jean-François Caron* \\ University of Houston \\ E-mail: jcaron@uh.edu
}

Mu2e is an experiment under construction at Fermilab that will search for the coherent neutrinoless conversion of a muon into an electron in the field of an atomic nucleus. This is a chargedlepton flavour violating process, and its observation would be clear evidence of physics beyond the standard model. Mu2e's single-event sensitivity of $3 \times 10^{-17}$ is 4 orders of magnitude better than previous experiments looking at this reaction, and indirectly probes energy scales beyond those envisioned for high-energy colliders. To achieve the high sensitivity requires a very intense but low-energy muon beam, a high-precision measurement of the conversion electron's momentum, and several key mechanisms to reduce background events. This work presents an overview of the experimental design and construction status, focusing on four "Big Deal" items which are particularly innovative or interesting.

XIV International Conference on Heavy Quarks and Leptons (HQL2018)

May 27- June 1, 2018

Yamagata Terrsa, Yamagata,Japan

${ }^{*}$ Speaker. 


\section{Introduction}

Lepton flavour is an accidental conserved quantity of the Standard Model of particle physics. There is no fundamental symmetry that requires it to be conserved. Moreover, the discovery of neutrino oscillations is direct evidence of non-conservation of lepton flavour. It is a natural question to ask if this is limited to the neutrinos, or whether the heavy leptons also experience flavour-nonconserving interactions. Mu2e will search for the decay of a nuclear-bound muon directly into an electron, with no neutrinos emitted.
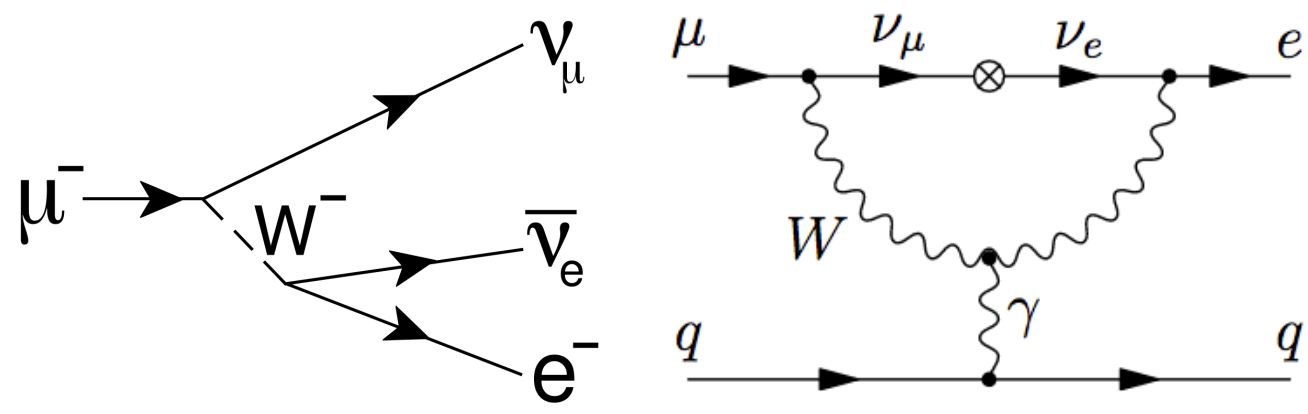

Figure 1: Feynman diagrams for regular muon decay (left) and the flavour-violating decay in the Standard Model with neutrino oscillations, with a quark from a nucleus participating (right).

In the Standard Model with massive neutrinos, this reaction can happen via the oscillation of a virtual neutrino in a loop. This process is however suppressed by the ratio $\Delta m_{v}^{4} / m_{W}^{4}$ and the branching fraction is calculated to be $\sim 10^{-54}[1]$. This is small enough to be completely unobservable, and makes the measurement essentially free from Standard Model backgrounds. A broad class of physics models beyond the Standard Model would enhance the branching fraction. A search is well-motivated by supersymmetry (SUSY) at the level of $R_{\mu e} \sim 10^{-15}$ [2] where the experimental variable is the ratio of flavour-violating direct decays to the usual muon capture:

$$
R_{\mu e}=\frac{\mu^{-}+A(Z, N) \rightarrow e^{-}+A(Z, N)}{\mu^{-}+A(Z, N) \rightarrow v_{\mu}+A(Z-1, N)} .
$$

For SUSY models with dimension 6 operators occurring in loops, Mu2e effectively probes mass scales of thousands of $\mathrm{TeV}$ for unit coupling; this is well beyond the reach of foreseeable collider experiments.

The current best limit is $R_{\mu e}<7 \times 10^{-13}$ from Sindrum II[3], which Mu2e will improve on by four orders of magnitude. Typical SUSY models used to explain electroweak symmetry breaking can predict $R_{\mu e} \sim \mathscr{O}\left(10^{-15}\right)$, in which case Mu2e would see roughly 40 signal events in 3 years of running.

Other than the lack of neutrinos (which are not detected even in the normal muon decay process) and the tiny nuclear recoil, the only observable difference in the final state is the momentum of the outgoing electron. Thus Mu2e needs a large number of stopped muons, extremely good momentum resolution, and extreme suppression of other sources of high-energy electrons. 


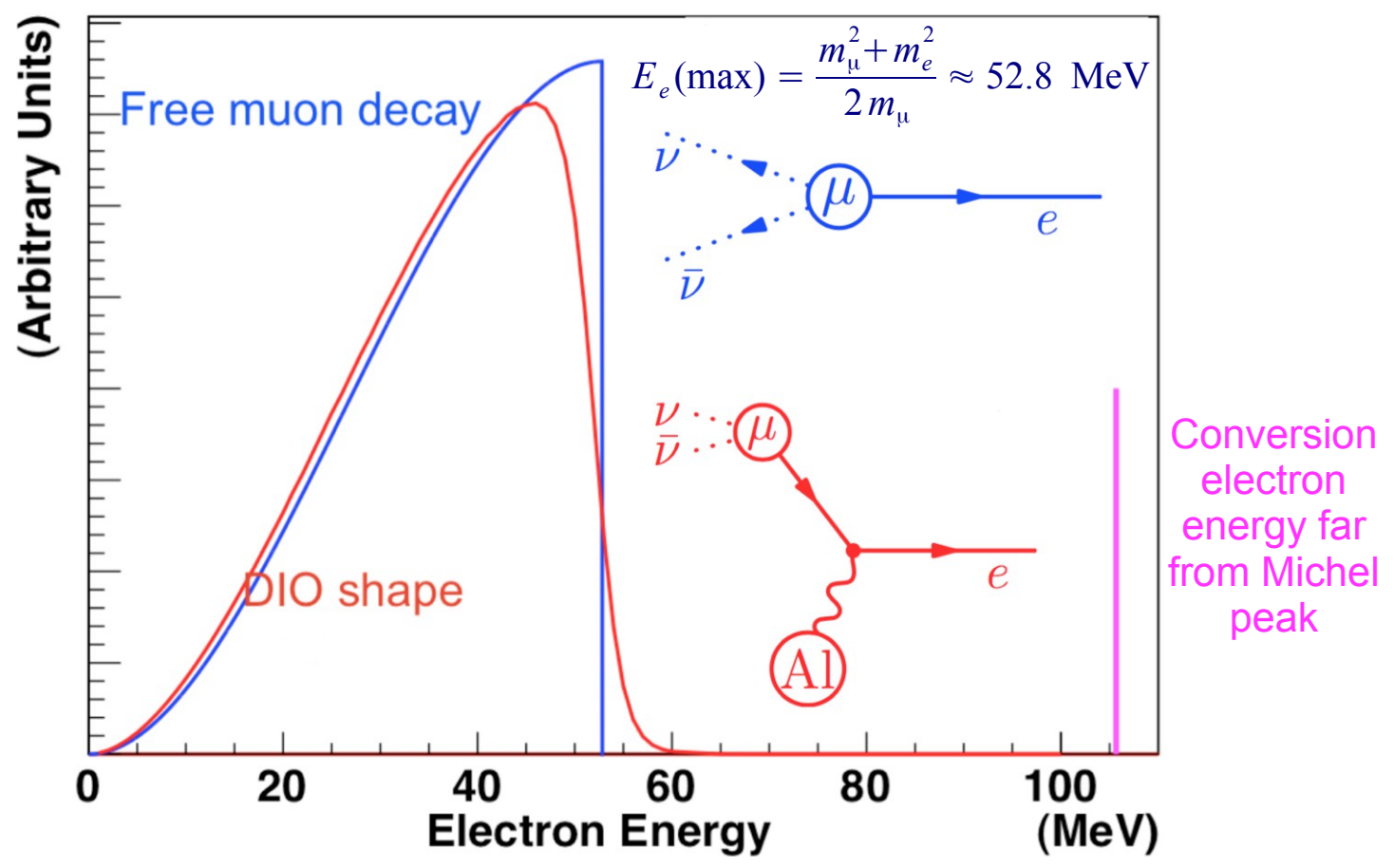

Figure 2: Spectrum of outgoing electrons from muon decays. In blue is the so-called Michel spectrum for a free muon decay, with a sharp endpoint at $52.8 \mathrm{MeV}$. In red is a muon decaying in orbit (DIO) around a nucleus. In magenta is the mono-energetic spike for a flavour-violating decay electron. The DIO endpoint is near the flavour-violating decay energy, and with detector resolution smearing, they do overlap.

\section{Big Deal \#1: Pulsed Beam}

Mu2e produces muons by colliding an $8 \mathrm{GeV} / \mathrm{c}$ proton beam from the Fermilab accelerator complex with a tungsten target. This produces many short-lived particles that decay into muons, notably pions. Sindrum II's result for $R_{\mu e}$ was limited by pion backgrounds, because it used a continuous proton beam.

A novel feature of Mu2e, originally proposed for the Muon Electron Conversion (MELC) experiment $[4,5]$, is a pulsed beam with enough time between pulses to allow pions to decay between signal windows. Mu2e uses a $1695 \mathrm{~ns}$-long period between proton pulses, with a signal window $700 \mathrm{~ns}$ after each pulse. Extinction is the ratio of the number of protons hitting the production target outside of the defined pulse width to the number of protons inside the pulse. An extinction of $\sim 10^{-10}$ is enough to make the pion contribution to our backgrounds acceptably small.

This high level of extinction is provided by a specialized AC dipole magnet that kicks protons out of the beam between each pulse.

\section{Big Deal \#2: B-Field Gradient and S-Bend Solenoid}

The second essential feature that makes Mu2e possible is the graded magnetic field around the production target. The proton beam is pointed away from the detectors in order to minimize 


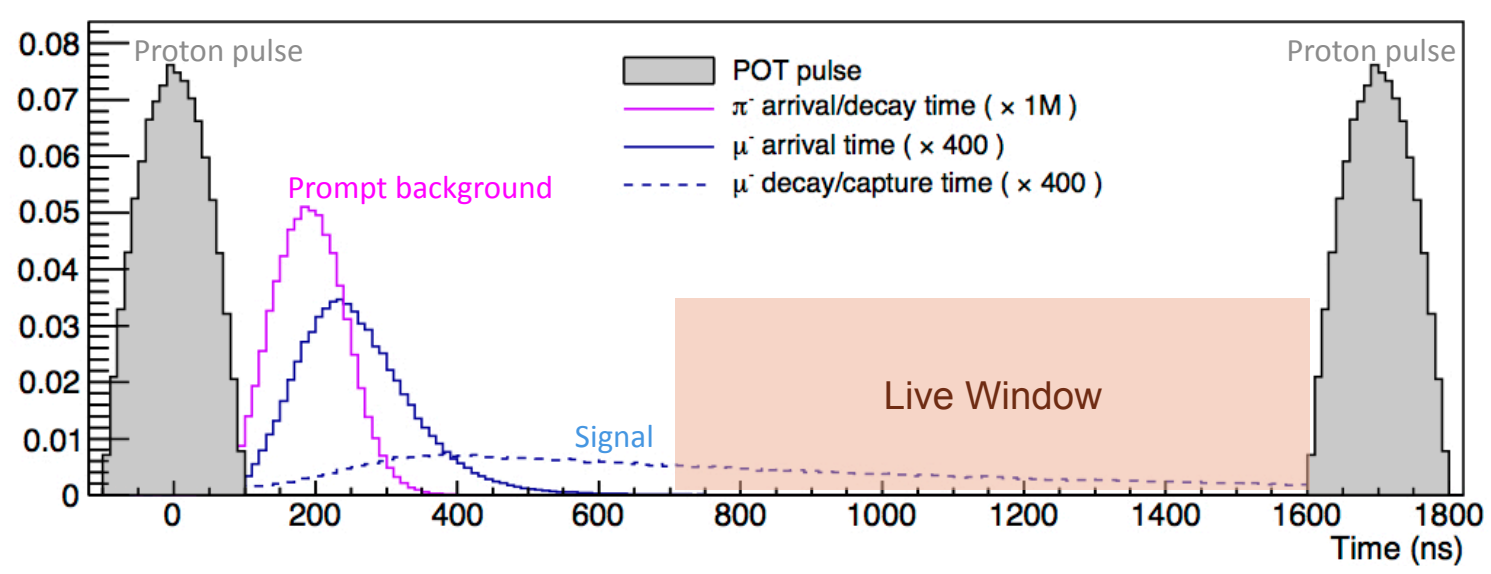

Figure 3: Time structure of the proton beam arriving at the production target. About $50 \%$ of the captured muon decays occur during the live window.

exposure to hadronic collision products, but the magnetic field gradient curves the lighter charged particles from the target back around towards the muon beamline, increasing the overall efficiency.

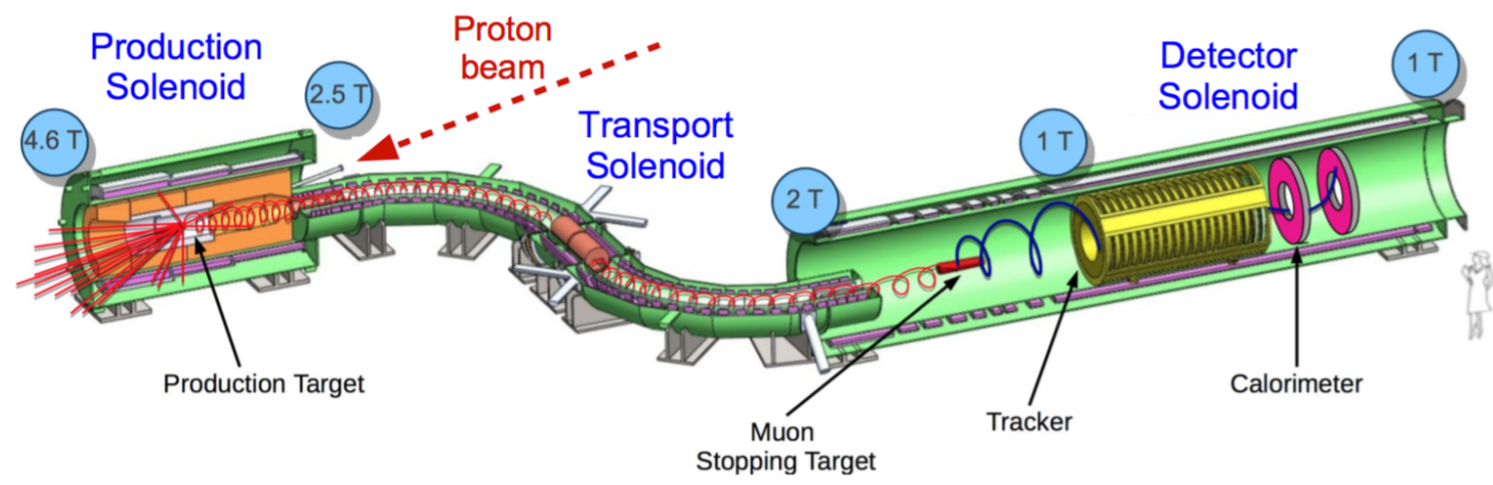

Figure 4: The three solenoids which make up the Mu2e muon beam line. Magnetic field amplitudes are listed at various points.

The proton beam dumps approximately $8 \mathrm{~kW}$ of power on the production target, which sits inside a vacuum vessel, inside a superconducting solenoid. The extremely high power density is limited by what is achievable with current engineering. Specialized machinery is being constructed to change out the production targets, which are expected to last about 1 year each. Large brass pieces sit between the solenoid and the vacuum vessel to act as a heat and radiation shield.

The particles from the proton collision that are directed backwards enter the S-shaped transport solenoid. This eliminates neutral particles and gives kaons and pions enough time to decay into muons. The curvature of the bends selects only the lower-momentum muons $(<80 \mathrm{MeV} / \mathrm{c})$ that have a high chance $(\sim 60 \%)$ to capture on the stopping target. A special collimator in between the bends selects only those muons with the correct charge. For every proton hitting our production target, we get 0.0019 stopped muons in the stopping target. The transport solenoid also has a magnetic field gradient to avoid trapping any particles that could later decay and produce background events. 


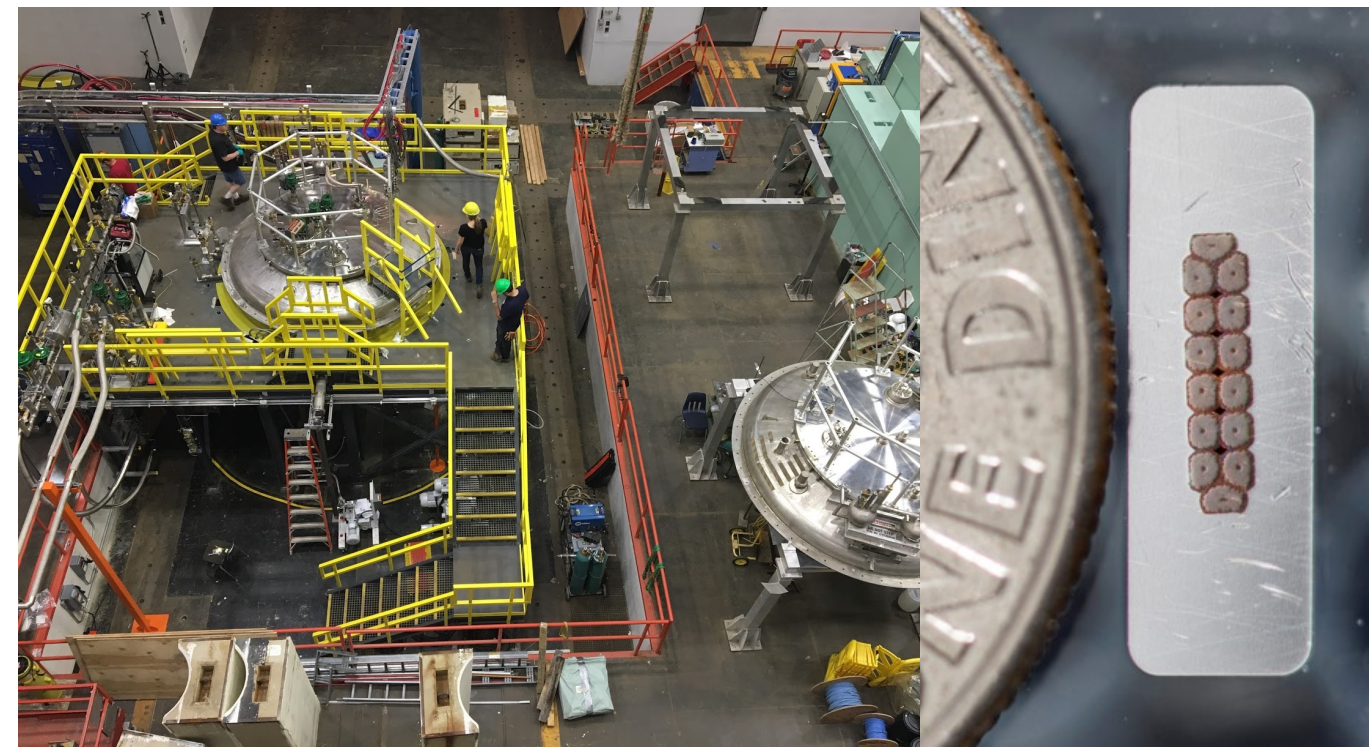

Figure 5: Left: Solenoid test facility at the Heavy Assembly Building (formerly CDF) at Fermilab. Two caps allow one coil to be tested while another is attached or detached from the test fixtures. Right: cross-section of the aluminum-stabilized NbTi superconductor.

All the superconductor for the solenoids has been produced and winding of the coils is ongoing. The first of the smaller transport solenoid modules have already been delivered to Fermilab. A full facility is operational at Fermilab where each module will be tested (see Figure 5).

\section{Big Deal \#3: A Holey Tracker}

As mentioned in the introduction, the only way to discriminate between electrons coming from a regular muon decay and a flavour-violating decay is the momentum. Thus we need a very high-resolution tracking detector. Momentum resolution is limited by multiple scattering of the electron in the detector material itself, so the key is to have an extremely low-mass tracker. The novel solution for Mu2e is a cylindrical straw tracker with a hole in the middle. Electrons from a regular muon decay have a momentum of $\sim 55 \mathrm{MeV} / \mathrm{c}$, so the radius of their helical tracks will mostly miss the straws, or only deposit partial tracks. Electrons from a flavour-violating decay will have much higher momenta of $104.97 \mathrm{MeV} / \mathrm{c}$ and their larger helices will cross the straw volume (see Figure 6).

All the readout electronics lie on the outside of the active detector volume, so electrons coming from a flavour-violating decay encounter $<1 \%$ of a radiation length. This allows us to reach a transverse momentum resolution better than $180 \mathrm{keV} / \mathrm{c}$.

The straws are wound from two layers of $12 \mu \mathrm{m}$ aluminized mylar. The inner layer also has a gold coating. The straws have all been purchased and wound. Assembly of the tracker modules and development of the readout electronics is ongoing. 

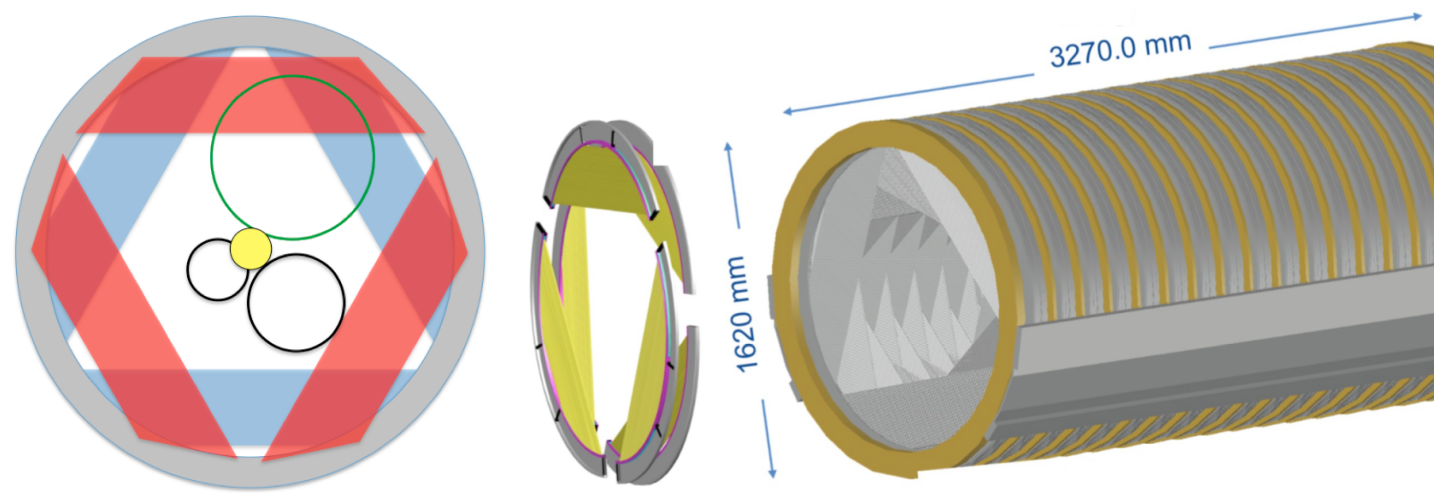

Figure 6: Left: Length-wise projection of electron tracks in the tracker. The yellow disc represents the stopping target from which the electrons will be emitted. The smaller black circles are helices taken by electrons from regular muon decay, while the larger green circle is taken by higher-energy electrons from flavour-violating decays. Right: Rendering of the fully assembled tracker.

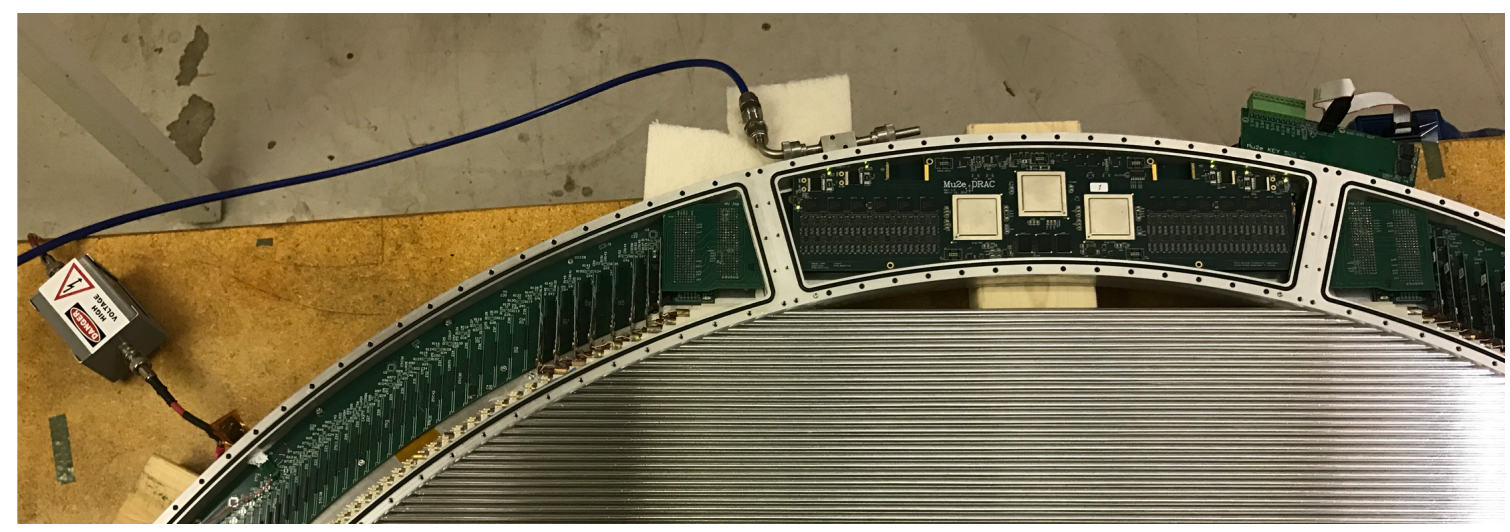

Figure 7: Prototype tracker module with covers removed, showing the electronics. The board on the left amplifies the analog signal and transmits it to the central board labelled "Mu2e DRAC" (Digitizer Readout Assembler and Controller).

\section{Big Deal \#4: Cosmic Ray Veto}

Our simulations show that without mitigation, cosmic rays would produce approximately 1 fake signal-like electron in the Mu2e detectors per day. Recall that with $R_{\mu e}=10^{-15}$ we expect to see 40 signal events in 3 years. Burial or other passive shielding being unfeasible, the only option is to detect every passing cosmic ray with high efficiency and veto the signal-like events that coincide with a cosmic ray hit. The area to be covered is $327 \mathrm{~m}^{2}$, and we require an efficiency of $99.99 \%$. The Mu2e cosmic ray veto (CRV) consists of $26 \mathrm{~km}$ of plastic scintillator with dual wavelength-shifting fibres inside, each read out by silicon photomultipliers. The expected signal loss from CRV dead-time is a few percent.

All the scintillator has been produced, late-stage prototypes exist for the fixtures, and procurement of the silicon photomultipliers (from Hamamatsu) and wavelength-shifting fibres is ongoing. 

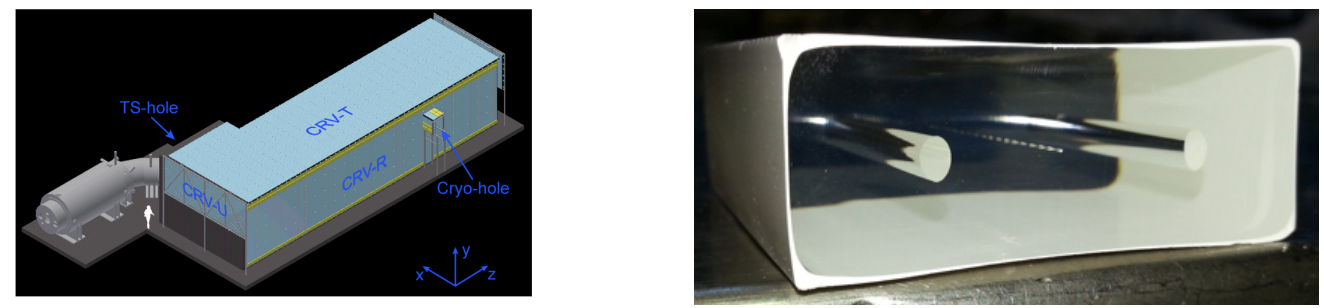

Figure 8: Rendering of the complete CRV (left) and a cross-section of the extruded plastic scintillator (right).

\section{Summary}

The Mu2e project has moved out of the design phase and is now in the early construction stage. We are on track to take commissioning data in 2022, with 3 years of physics data after that. Several essential components of the project were not reported on here for the sake of brevity; the interested reader is referred to the presentation slides from HQL2018 and to the publicly-visible document page on the Mu2e website[6].

Initial discussions have already taken place for an eventual upgrade to Mu2e, with an expression of interest submitted to the Fermilab leadership[7].

\section{Acknowledgements}

The speaker acknowledges the support of the DOE Office of Science through grant DESC0011598 to the University of Houston.

We are grateful for the vital contributions of the Fermilab staff and the technical staff of the participating institutions. This work was supported by the US Department of Energy; the Istituto Nazionale di Fisica Nucleare, Italy; the Science and Technology Facilities Council, UK; the Ministry of Education and Science, Russian Federation; the National Science Foundation, USA; the Thousand Talents Plan, China; the Helmholtz Association, Germany; and the EU Horizon 2020 Research and Innovation Program under the Marie Sklodowska-Curie Grant Agreement No.690835. This document was prepared by members of the Mu2e Collaboration using the resources of the Fermi National Accelerator Laboratory (Fermilab), a U.S. Department of Energy, Office of Science, HEP User Facility. Fermilab is managed by Fermi Research Alliance, LLC (FRA), acting under Contract No. DE-AC02-07CH11359.

\section{References}

[1] Mu2e Collaboration, "Mu2e Technical Design Report," Fermilab, October 2014. Mu2e Document Database Link

[2] L. Calibbi and G. Signorelli, "Charged Lepton Flavour Violation: An Experimental and Theoretical Introduction,” Riv. Nuovo Cim., vol. 41, no. 2, p. 1, 2018.

[3] W. Bertl, R. Engfer, E. Hermes, G. Kurz, T. Kozlowski, J. Kuth, G. Otter, F. Rosenbaum, N. Ryskulov, A. van der Schaaf, P. Wintz, and I. Zychor, "A search for $\mu$-e conversion in muonic gold," The European Physical Journal C - Particles and Fields, vol. 47, pp. 337-346, Aug 2006. 
[4] R. Dzhilkibaev and V. Lobashev, "On the seach for Mu-e conversion on nuclei. (In Russian)," Sov.J.Nucl.Phys., vol. 49, pp. 384-385, 1989.

[5] V. S. Abadzhev et al., "MELC experiment to search for the $\mu^{-} A \rightarrow e^{-} A$ process," 1992.

[6] Mu2e public results page. http://mu2e.fnal.gov/public/hep/results/index.shtml

[7] Mu2e Collaboration (F. Abusalma et al.), "Expression of Interest for Evolution of the Mu2e Experiment," 2018. ArXiv Link 